A conferência que publicamos a seguir foi proferida por Inês Oseki-Depré, em setembro de 2009, na Casa das Rosas, em São Paulo, durante o II Encontro de tradutores de obras francesas no Brasil, organizado por docentes da USP no âmbito das atividades do ano da França no Brasil.

Inês Oseki-Depré é tradutora e professora de Literatura Comparada da Universidade da Provença, no sul da França. Brasileira, estudou na USP nos anos 1960 e fez seu doutorado na França, onde se estabeleceu. Tem trabalhos importantes sobre a tradução literária - em especial Traduction et Poésie (dir., Maisonneuve \& Larose, 1996), Théories et Pratiques de la traduction littéraire (Armand Collin, 1999) e De Walter Benjamin à nos jours (Honoré Champion, 2006) - e longa prática como tradutora, do francês para o português - realizou, por exemplo, a primeira tradução para o português dos Escritos de Jacques Lacan -, e sobretudo do português para o francês - traduziu José de Alencar, Guimarães Rosa, Ligia Fagundes Telles, Antonio Vieira, Carlos Drummond de Andrade, Fernando Pessoa, Haroldo de Campos (sua tradução de Galáxias ganhou o prêmio Roger Caillois do melhor livro estrangeiro em 1999). Nos últimos anos tem atuado como professora visitante em algumas universidades brasileiras e é gestora, junto à Universidade da Provença, de convênio entre o Centro de Pesquisa em Literatura Geral e Comparada e em Tradução Literária, dessa universidade, e o Programa de Pós-Graduação em Letras Neolatinas da UFRJ.

A conferência aqui apresentada é uma reflexão sobre sua tradução do livro Quelque chose noir (Algo: preto), do poeta contemporâneo francês Jacques Roubaud, que Inês publicou em 2005 pela editora Perspectiva, em coleção dirigida por Haroldo de Campos. Inês apresenta as dificuldades de transposição de toda a memória poética de Roubaud para a língua portuguesa, trabalho que, como conclui o ensaio, faz surgir "em nossa língua uma poética inédita, herdeira de formas e tradições diversas, ausentes em nossa memória”.

Marcelo Jacques de Moraes (UFRJ) 


\section{D'UNE TRADUCTION AMNÉSIQUE (À PROPOS DE Algo: Preto, de Jacques Roubaud)}

Inês Oseki-Dépré
Dans "Quelques thèses sur la poétique", Jacques Roubaud affirme: "toute littérature est mémoire, et code, d'une langue, et du langage". C'est la thèse centrale de sa poétique.

Par ailleurs, comme l'a dit quelqu'un (peut-être Ponge?), "le poème est étranger dans sa langue", ce qui en constitue l'antithèse.

Il s'agit d'examiner l'œuvre roubaldienne sous cet angle et de commenter la cohérence de sa poétique, en particulier en ce qui concerne Quelque chose noir, paru en 1986. Est-il tributaire d'une tradition? Est-il simplement un poème roubaldien? Qu'en est-il de sa traduction brésilienne?

Je commencerai par une brève présentation de l'auteur, qui sera suivie d'un résumé de sa poétique (ses œuvres) et terminerai par l'œuvre en question et sa traduction portugaise (brésilienne).

J'essaierai ainsi d'envisager le texte original de Algo: Preto, en français Quelque chose noir, dans son rapport à la langue, à la tradition et à la rupture, et d'y apporter quelques réflexions concernant ma traduction.

\section{Introduction: Jacques Roubaud et son œuvre}

Ma présentation de Jacques Roubaud sera brève. Brève pour respecter le temps de ma conférence, mais aussi parce je tenterai d'aller à l'essentiel, étant donné l'immensité de la personne du poète et donc de son œuvre.

Considéré actuellement comme l'un des poètes les plus importants de la littérature française contemporaine, Jacques Roubaud, né en 1932 dans le sud-ouest de la France, est à la fois poète, traducteur, mathématicien, et a été jusqu'à date récente directeur d'études à l'Ecole de Hautes Etudes en Sciences Sociales après être passé par l'Université de Paris X, comme professeur de mathématique.

Marqué par une éducation ouverte à la philosophie, à l'histoire, à la littérature, à l'entomologie... Jacques Roubaud a été pris très tôt, selon sa fille Florence, par le "ice cube syndrom", hérité de sa grand-mère, qui consiste à ne pas parler de soi ni de ses sentiments.
(ROUBAUD, Jacques "Quelques thèses sur la poétique". Change, n.6. Paris Seuil, 1970: 12) 
- (Banana Split, no. 16, oct.déc. 1985/janvier 1986.)

- (Les Troubadours. Paris: Seguers, 1971.)

(Graal théâtre avec Florence Delay. Paris: Gallimard, 1977; Graal fiction. Paris, Gallimard, 1978.)

- (La Fleur Inverse, étude sur l'art formel des troubadours. Paris: Ramsay, 1986.)
Comme d'autres grands poètes (Borges, Pessoa, Baudelaire), il a publié ses premiers poèmes à l'âge de 12 ans (Poésies juvéniles) mais son premier livre important, Signe d'appartenance, date de 1967, début d'une phase "japonaise". Mono no aware date de 1970.

Coopté par l'Oulipo (Ouvroir de la Littérature Potentielle) en 1966 sur proposition de Raymond Queneau et François Le Lionnais, Jacques Roubaud a toujours associé une grande rigueur formelle à un profond savoir de la littérature française et étrangère, dont il fut également traducteur et dont témoigne grande part de son œuvre. Sur le versant oulipien, on peut citer Autobiographie chapitre $X$ (1977), dans laquelle le poète démonte et recrée des poèmes de Reverdy, Cendrars, Desnos, Eluard. Ce procédé sera utilisé également, entre autres, dans Voyage d'Hiver, écrit après Georges Perec (1997). Co-fondateur de l'Alamo avec Paul Braffort, il a ouvert en 1989 avec le Grand Incendie de Londres un long cycle de prose, entreprise qui constitue son "projet, regardé et raconté par [lui]". Inventeur de plusieurs contraintes (dont le "baobab" et le "haïku oulipien généralisé"), il est l'auteur du premier "voyage", suite du Voyage d'hiver perecquien, le Voyage d'hier, préparant la voie au premier roman collectif de l'Oulipo.

A côté de cette formation mathématico-oulipienne, une autre source de sa poésie provient des troubadours occitans, poètes qui associent le thème de l'amour et de la femme à un formalisme tout aussi important, qu'ils imbriquent à la musique et auxquels le poète est attaché pour des raisons territoriales. Dans un entretien, ${ }^{*}$ Roubaud explique sa relation à Ezra Pound, autre amoureux de la poésie troubadouresque, mais dont l'un des apports principaux pour lui a été de lui faire découvrir les poètes du groupe Noigandres... Sur les troubadours, ce dernier propose une précieuse anthologie bilingue, en plus du le cycle du Graal," dans lequel les aventures des héros sont racontées selon plusieurs schémas narratifs est construit sur des textes de Gauvain Lancelot, Perceval, Guenièvre, et une étude.

Des essais sur le vers, sur l'alexandrin (La Vieillesse d'Alexandre, 1978), sur le sonnet en particulier (Soleil du soleil, 1990), sur la mémoire (L'Invention du fils de Léoprépès, 1993), sur la poésie en général (Poésie, etcétéra: ménage, 1995, La ballade et le chant royal, 1998), parsèment cette œuvre riche et volumineuse dont je ne donne que quelques exemples.

En tant que poète, son œuvre contient des textes poétiques en vers ou en prose narrative. Des premiers sont connus Mono no awa- 
re (1970), Trente et un au cube (1973), Quelque chose noir (1986), La Forme d'une ville change plus vite, hélas, que le coeur des humains (1991-1999), une anthologie Churchill 40 et autres sonnets de voyage (2004), 128 poèmes (2006). La plupart de ses poèmes en vers ou en prose et de ses récits sont constitués de textes dont beaucoup à contraintes formelles, comme la trilogie des Hortense (La Belle Hortense, 1985, L'Enlèvement d'Hortense, 1987, L'Exild'Hortense, 1990), ou Le Grand Incendie de Londres (1989), La boucle (1993), plus récemment La Bibliothèque de Warburg (2002), Parc Sauvage (2008) et Impératif catégorique (2008). C'est dire que le poète assure une production continue et variée. Au Brésil, pourtant, il est peu traduit.

On ne pourrait finir cette énumération partielle sans évoquer ses travaux de traduction, qui s'insèrent à l'intérieur de sa production in progress suivant la même trajectoire, le même projet. Comme traducteur, en effet, son premier travail a été fait en collaboration avec d'autres poètes. Renga (1971) a été traduit/ recréé du japonais en collaboration avec Octavio Paz, Edoardo Sanguinetti, Charles Tomlinson, le renga étant une forme poétique collective (apparue durant la période Heian 794-1192), dotée comme le dit Claude Roy "d'une extrême et complexe rigueur". Claude Roy ajoute des remarques sur ce travail mû par "le goût commun des formes composées, des règles du jeu combinatoires" qui laissent néanmoins à chacun "ce jeu qui signifie à la fois la liberté, l'aisance de mouvement et la rigueur des règles", bref, comme le dira Jacques Roubaud, "le sentiment de l'unité changeante". Roubaud lui-même l'explique, dans sa description de cette tentative, et nous verrons que c'est fondamental pour la compréhension de son œuvre:

La liaison des chainons, des strophes, en un tout qui est le renga, poème de poèmes, est soumise à des règles destinées à assurer que le double mouvement simultané de continuité et de rupture est conforme à l'esthétique de la forme.

Le masterpiece à huit mains a été suivi plus loin d'une traduction à plusieurs mains avec Michel Deguy, entre autres, autre grand nom de la poésie française. Ainsi, en 1981, apparaît Vingt poètes américains, en édition bilingue dirigée par les deux poètes. Dans cette année aussi, est publiée sa fameuse traduction du Snark, de Lewis Carroll, autre mathématicien, en sizains.

En 1988, avec Florence Delay, il publie Partition rouge (traduction de poèmes des indiens d'Amérique). En 2000, il participe à une traduction collective de la Bible, connue comme la "Bible 
Bayard", d'où se détache particulièrement une traduction du Qohélet, traduit aussi en portugais par Haroldo de Campos.

La traduction apparaît ici comme un exercice poétique, intégrant une pratique également créatrice ("transcréation" dirait Haroldo), dans laquelle on retrouve les mêmes principes ou programme: tradition et rupture, rigueur formelle, dialogue entre ancien et moderne (le "makeitnewisme", comme il le dit). Traduction et écriture se complètent, faisant partie d'une même attitude commune, où l'histoire joue un grand rôle, l'histoire comme fond sur lequel s'enchaînent évènements et textes.

\section{Une poétique originale}

L'histoire, le politique, le lien social n'ont, en effet, jamais été absents du projet roubaldien. Dès les années 1968, avec d'autres poètes, Jacques Roubaud fait partie du collectif Change, revue caractérisée par un esprit avant-gardiste (le comité est constitué de Jean-Pierre Faye, Jean-Claude Montel, Jean Paris, Léon Robel et Jacques Roubaud).

En 1970, initiateur du volume La poétique, la mémoire, il propose ses thèses sur la poétique qui inscrivent la poésie dans le fonds commun, la langue. Reprenant sa réflexion à un travail présenté au cercle Polivanov, dont il est l'un des fondateurs (Cercle de poétique comparée) en mai-juin 1969, s'appuyant sur les derniers travaux de Chomsky, du Barthes du Degré Zéro, de John Thompson, de Meschonnic (avec qui il sera brouillé plus tard), Roubaud propose comme hypothèse le rapport intrinsèque entre le discours de l'écrivain et la littérature (Barthes), le discours de l'écrivain et le langage (Thompson). Bref, la littérature parle du langage tout en parlant d'autre chose, mais la littérature parle "de ce que le langage dit d'autre chose, du ce que et du comment."

C'est ainsi qu'il en vient à dire que la littérature est mémoi-

(ROUBAUD, Jacques. "Quelques thèses sur la poétique". op. cit.: 12.) re, et code, non seulement d'une langue particulière, mais aussi du langage, de son fonctionnement, de ses lois.. Par mémoire, faute de mieux, il désigne "tout ce qui est préservation, détermination sémantique" (le "ce que"); par code, "ce qui est préservation, détermination des lois formelles, à différents niveaux, du langage (sons et phrases, rythme, discours, etc., le "comment")".

La littérature "parle de cette langue dans cette langue", en fixant son originalité, ses traits particuliers, ses sons, sa syntaxe; tou- 
tefois elle "tendra toujours à rendre compte de ce qui, dans l'objet langue, a changé, parce que du nouveau tend à apparaître". *

Bref, le rapport entre littérature et langage est un rapport nécessaire, l'un n'existe pas sans l'autre, le langage vit de la mémoire de la littérature. Les modifications, les innovations littéraires permettent la survie du langage tout en le préparant à de nouvelles lois, tout en ouvrant le chemin à de nouvelles découvertes conceptuelles.

On voit ici la théorisation de sa pratique, qui le rapproche des grands poètes: le regard vers la tradition, vers le fonds littéraire dans sa langue et dans d'autres langues, par rapport auquel la poésie opérera des ruptures, dans laquelle la poésie introduira des formes nouvelles, modifiant la langue et ouvrant de nouveaux territoires de la pensée. Ce qui s'observe aisément dans son travail de poète et de traducteur.

D'abord les formes japonaises, le renga en particulier, régi par les shikimokué: prescriptions formelles relatives au vocabulaire, aux formes grammaticales accompagnées de prescriptions thématiques (choses ou concepts dont il est donné une liste). Sa règle la plus importante est donnée par le sarikiren, continuité et rupture: thème saisonnier (trois ou cinq strophes s'il s'agit du printemps ou de l'automne, trois s'il s'agit de l'été ou de l'hiver).

Les troubadours ensuite, ancêtres en quelque sorte de ce poète occitan, et on verra que cette source n'est pas étrangère à notre poème. On peut lire dans l'introduction à son anthologie:

L'amour ("premier principe" qui gouverne le monde) inspire le chant, et le chant trouve son incarnation la plus élevée la plus "fine" (pure), dans la canso, qui chante l'amour, qui est le lieu, la chambre de l'invention, poésie [...] l'art triple-un du trobar est l'unité parfaite à laquelle aspire le troubadour, à la fois don imposé par l'amour, inspiration et métier inexorable, exigeant, tous deux nécessaires pour fabriquer, forger, limer, l'objet sonore, poétique et musical, qui chante l'amour.

Après la description des caractéristiques principales de cette poétique: le lieu (Proensa), les principaux troubadours et ses dames, les thèmes des amors, la beauté de la dona, le regard, les sentiments (dezir, jö̈, sofrirs), la mezura, la merce, Jacques Roubaud définit la canso, le poème: constitué sur la strophe, lieu où le poème s'élabore, dont le troubadour est l'architecte. La formule de la canso est triple: elle concerne la métrique, les rimes et la mélodie. Il fait remarquer la grande richesse de la versification troubadouresque: entre une et 14 syllabes suivant plusieurs schémas, avec ou sans césure, avec des 
vers rimés, parfois au moyen de la rime riche ou de la rime "hirsute" (voir allusion dans Quelque chose noir), celle dont la sonorité est heurtée, explosive, sèche qui s’oppose aux grandes sonorités douces (rimes caras). Parfois on retrouve le même mot répété à la rime.

Toute cette combinatoire produit un jeu rythmique d'une grande complexité et d'une grande variété. Selon Istvan Frank, cité par le poète, il existerait 900 types de rimes différents sur 2600 textes. L'organisation des poèmes est tout aussi complexe: chaque cobla d'une canso a la même formule qui détermine pour chaque chant la mélodie et la disposition des mètres et des rimes. On va retrouver donc des cansos unisonans, dans lesquelles les coblas seront capfinidas (reprise dans une strophe en dehors de la première d'un mot du dernier vers de la précédente), capcaudadas (reprise de la dernière rime). En somme, trobar est un métier. Traduire les troubadors un autre, que Jacques Roubaud partage avec Augusto de Campos.

L'autre composante de la poétique roubaldienne est, on l'a dit, la mathématique. Le goût de la rigueur, de la formule, des codes se retrouve satisfait dans ce courant poétique, fait d'invention et de liberté (chaque œuvre possède ses propres contraintes).

\section{Quelque chose noir/ Algo: Preto}

Qu'en est-il de Quelque chose noir? Et, par conséquent, de Algo: Preto?

En fait, Quelque chose noir fuit en quelque sorte la règle. C'est une œuvre d'exception, à commencer par le titre. Ecrit en 1986, après la mort prématurée de sa femme, la photographe Alix Cleo Roubaud, et malgré le soin prosodique et la haute teneur poétique des poèmes qui le composent, le livre dépasse la question des procédés et des règles de construction - tout en les maintenant pour atteindre un très haut degré de création dans le désir de dire la mort de la personne aimée.

Dans un entretien accordé à Manuel da Costa Pinto, pour la Folha de São Paulo du 16 avril 2005, Jacques Roubaud s'exprime:

"Les poèmes de ce livre portent les vestiges d'un effort pour vaincre le silence imposé par la mort. La parole rythmique, inscrite dans la poésie et dans le vers, était pour moi un signe de vie. Devant la mort, toutefois, j’ai été frappé par une incapacité de parler

•(Cf. le poème "Afasia". Algo: Preto. Coleção Signos. São Paulo: Perspectiva, 2005: 130.) de poésie, par une aphasie.”

En tant que traductrice et lectrice de Roubaud, je l'ai choisi parce que justement il se présentait sous une forme assez libre de 
contraintes (qui auraient nécessité une réélaboration hypertextuelle pas toujours aisée en portugais, moins pour la continuité paradigmatique que pour la continuité chez un seul poète, les frères Campos ou Décio Pignatari constituant une exception), donc assez traduisible, et parce que j'ai été très touchée par le texte qui parle de la mort. J'ai rencontré peu de difficultés, mais quelques-unes de taille. Je commencerai par le titre "Quelque chose noir", qui, comme j'ai déjà eu l'occasion de le dire, est pratiquement agrammatical en français, où il aurait fallu dire "quelque chose de noir" ou "quelque chose noire" au féminin. Haroldo de Campos, mais avant lui Nelson Ascher, avaient suggéré "negro" qui, comme on le sait, est plus "littéraire", plus "noble" en portugais. J'ai tenu tête, "noir" fait partie de l'expression française "en noir et blanc" pour parler de la photo (comme dans la chanson Retrato em branco e preto...). C'est alors que Haroldo de Campos (directeur de la collection) a proposé qu'on ajoute les deux points, pour qu'on maintienne l'étrangeté, ce qui a entrainé en portugais "Algo: preto". Ces deux points ouvrent la signification du recueil vers quelque chose qui va arriver, créent une sorte de suspense. Pour le reste, parfois j'ai accentué un peu plus la signification d'un mot, "noirceur" traduit par "preto" et non "pretura", "encombrements", traduit par "excessos", et non "entulhos"...

La mort est vacuité, solitude, perte. Dans ce poème en neuf sections, le poète tente d'exprimer la sécheresse du vide comme à travers des instantanés (d'où le "noir et blanc"), adoptant le ton de l'immobilité suggestive du photographe et en dialogue avec l'œuvre de sa femme, Alix, photographe, pour fixer en même temps le néant, l'irréversible, mais captant simultanément l'instant où de deux il ne reste qu'un qui souffre.

Pour Jacques Roubaud, ce poème n'offre pas de solutions de continuité évidente avec son œuvre, bien que bâti sur une série de contraintes autour du chiffre neuf, qu'il associe au deuil. On se rappellera l'importance du chiffre neuf pour Borges qui, dans la nouvelle "La demeure d'Astérion" ("La casa de Asterion"), associe le chiffre neuf à l'infini.

Or, cette clé, la formule "neuf", qui apparaît dès le départ, dans le premier poème de la première section "Méditation du 12/ 5/ 85" est restée intraduisible en portugais:

"Cette image se présente pour la millième fois à neuf”. * Ce mot polysémique en français ne l'est pas en portugais, et sa traduc-

(ROUBAUD, Jacques. Quelque chose noir. Paris, Gallimard, 1986: 11.) 
tion pose problème. On a vu qu'il indique la structure du poème, de la "neuvine"... Ma proposition a été:

"Essa imagem se apresenta pela milésima vez novamente", où "novamente" ne traduit pas tout à fait le mot "neuf", qui signifie "novo", "nove" et ici peut signifier "à nouveau", "de novo", "novamente", et, qui plus est, rime avec "veuf” (viúvo). L'allusion au "neuf" apparaît également dans "Vou me afastar", sous forme de multiple (3 fois 3 fois 3 ):

São três vezes tu três dos irredutivelmente separados deslocados reais de ti perdidos numa diáspora que une somente esse pronome: $t u$

Dans tout l'ouvrage, par ailleurs, les formes et les tons varient, fragments, visions, pensées et la progression suit le processus de la disparition: le corps mort, les souvenirs, la fin de la mort, la fin ("Quand ta mort sera finie, je serai mort", "Quando tua morte acabar, estarei morto"). De la première à la deuxième partie, aux questions lancinantes (“où es tu?”, "onde estás?”), au constat de la vacuité excessive du quotidien (voir le poignant poème sur la préparation du bol de café), d'une douleur inégalable, l'image de la femme se dessine, est présente, nue, pour atteindre une dynamique dans la section suivante sous forme de roman-photo où la photo est toujours présente, comme un leitmotive, une obsession qui relie les mots à l'image (en noir et blanc).

Le sofrir des Troubadours, l'évocation de Dante et Pétrarque survolent le poème, devenu "hirsute" en partie IV, mais la logique, Wittgenstein, apparaît ici dans une intertextualité pas forcée. La mort, si présente dans le livre, sera, par ailleurs, évoquée dans Le Grand Incendie de Londres (1985-1987), belle prose du grand troubadour aux accents japonais. Mais peu à peu la mort traverse le poème et les mots, et, de noire, devient opaque, devient blanche et claire, lumière, jusqu'à la mort du poète, qui s'est vidé jusqu'à la non-vie, Orphée revenant seul des enfers, puis "rien”, un rien qui, à l'instar de Fernando Pessoa, est tout.

Des pièces, par conséquent, où le soin, l'attention prêtée au dire se répètent et s'équilibrent avec l'amour, la complicité avec la femme aimée et les instants vécus, jamais pathétiques et cependant si précis comme une douleur aiguë, étonnants, dont la forme ciselée produit une grande émotion esthétique et affective: "Quelque chose noir qui se ferme et se tait, une déposition pure, inachevée" ("Algo preto que se fecha e se cala, uma deposição pura, inacabada"). 
Inachevée comme la mort, qui dure toujours. Ceci pour la mémoire, pour le "ce que" dit le poème.

Mais qu'en est-il du code? que dit le poème de la langue et de la poésie?

On peut rappeler ici que si l'attribution d'un chiffre à une idée provient de Pythagore, la neuvine est déjà la forme utilisée par Roubaud pour chanter Pétrarque ("Tombeau de Pétrarque"), sorte de sextine amplifiée, utilisée par Dante, Pétrarque et Arnault Daniel et reprise par Raymond Queneau (la sextine oulipienne, la "quenine"). Ce qui fait que si le vers est libre, la forme n'est jamais prosaïque. Ces indications, en plus de la "contrainte" de la neuvine, variante de la sextine, qui comporte ici neuf parties, chacune comportant neuf poèmes, nous font penser que même si le poète n'en a pas le projet, sa mémoire rappelle aussi bien l'amour, la perte de la femme aimée, que son souvenir de la poésie. Pour Stéphane Baquey, critique roubaldien, le livre est ainsi "une transposition formelle du sonnet de méditation compte tenu de la destruction de la tradition et de l'apport de poètes américains comme Gertrude Stein et David Antin". Il ajoute: "ainsi la forme du livre n'est plus trente un au cube, mais neuf au cube (à l'instar du Grand Incendie de Londres, commencé en 1985)", rappelant les sextines de Pétrarque. Ici, mémoire de la langue et mémoire du poète ne font qu'un: sa mémoire de poète lui rappelle la poésie et la langue dans laquelle il écrit, la poésie dans la langue dans laquelle il écrit.

Benoït Conort, poète, autre critique, consacre tout une partie de son article "Tramer le deuil” à la question des séries et des trous dans l'œuvre roubaldienne. Pour lui, "il faut rappeler que le chiffre est aussi architecture parce qu'il porte sens autre que mathématique, parce qu'il est mot et donc code chiffré". Il fait remarquer que si le jeu avec les chiffres fait partie du programme oulipien, chez Roubaud, dans le recueil qui nous concerne, il joue avec l'arbitraire, tout en en faisant une clef, ce qui fait penser qu'il fonctionne plutôt ici comme trompe-l'œil. Ceci entraîne comme conséquence que, pour le critique, "la contrainte se trouve confrontée à ses propres limites", poussant et freinant le mouvement à la fois. Pour preuve, elle apparaît en opposition à millième ("Cette image se présente pour la millième fois... à neuf avec la même violence..."। "Essa imagem se apresenta pela milésima vez... de novo com a mesma violência"), signifiant à la fois l'arrêt de quelque chose qui se répète, une "fin double": la fin de la femme aimée, et la fin du silence.
* Voir la thèse : Possibles de la poésie: Michel Deguy, Denis Roche, Jacques Roubaud, dir. Jean-Claude Mathieu, Université Paris 8 2006, à paraitre en $2010 \mathrm{aux}$ éditions Classiques Garnier.

"(CONORT, Benoït. "Tramer le deuil". La Licorne. Revue de langue et littérature française. Presses universitaires de Rennes, 2006: 2.) 
- (Stéphane Baquey, pp. 630-631)

•(CONORT, Benoït. "Tramer le deuil". op. cit.: 4.)
Le recueil est donc à la fois bâti sur le "neuf" et sur la répétition. Il créé sa propre structure, n'est plus mémoire de la langue.

Répétition du chiffre-nom, répétition des titres des poèmes à l'intérieur des neuf séries mais aussi répétition de vers, de fragments ou de mots, répétition des blancs (la mise en page).

Ainsi, au début du recueil nous rencontrons une méditation ("la méditation est un vice solitaire", dit Paul Valéry), dont la signification philosophique et religieuse désigne le recueillement mais aussi l'étude, la réflexion, qui se poursuit sur deux mois de l'année 1985. La seconde partie reprend la méditation durant le mois de la première méditation (capfinida?). La quatrième partie revient sur la méditation mais cette fois à travers l'intermédiaire du "portrait" ("Portrait de méditation", iii, iv, v), mais elle revient trois fois en $\mathrm{V}$ partie, partie centrale du recueil, pour ne plus revenir.

Les titres répétés sont significatifs. On a évoqué la "méditation" comme une forme de "médecine" chez les Indiens d'Amérique. Stéphane Baquey fait remarquer qu'elle rejoint la pratique poétique de Gertrude Stein (Stanza in Meditation) et de David Antin (Meditations), "sorte d'exercice logique".

On peut insister aussi sur la répétition du "roman-photo", qui attire l'attention sur le leitmotive du livre, sur la photo, sur le noir et blanc, l'image de la photo, la photo de l'image, sur laquelle on reviendra. Conort souligne l'importance de ce qu'il rappelle la tentation du roman, de la prose, contre laquelle le poète travaille. Il souligne également l'importance des répétitions accompagnées de variations et dans ce sens, dit-il:

Le livre, cette déposition (à prendre aussi dans le sens d'un témoignage, comme on fait une déposition auprès d'un tribunal, par exemple), s'accomplit à l'intérieur d'un mouvement contradictoire, la boucle, qui revenant à son point de départ donne l'impression de ne pas avancer; le dernie poème c'est encore le premier qui revient, et c'est toujours le même...

Comme dans C.R.A.Po.Po (en français C.R.A.Pi.Po.):

Eu olhava para ti. o escuro. o preto. o preto posto no ponto vivo. de teu ventre.

Eu batia o pé na relva. os doze pombos se alçavam um metro acima e depois se pousavam de novo.

Eu batia o pé na relva. os doze pombos se alçavam um metro acima e depois se pousavam de novo. 
Eu olhava para ti. o escuro. o preto. o preto pousado espesso no ponto. vivo. de teu ventre. $(. . .)^{*}$

*(ROUBAUD, Jacques. Algo:

De même le critique fait-il remarquer le lien entre Quelque chose noir et le reste de l'œuvre de Jacques Roubaud. Encadré par Dors, Les Tombeaux de Pétrarque, où le poète puisera la forme (proche aussi de l'anthologie des troubadours) et par Le Grand Incendie de Londres (roman), il appelle également La Pluralité des mondes de Lewis ("Echanges de la lumière"), La Boucle, ce qui lui permet de penser que notre recueil représente la pierre de touche de l'œuvre roubaldienne, celle qui va la réorienter, dans ce grand tissage. Par ailleurs, mis à part cet élément métalinguistique essentiel, le recueil, d'avoir été provoqué par une situation personnelle, la mort de la femme aimée, de l'aveu de Jacques Roubaud lui-même, est exempté de contraintes ("restriçōes") intraduisibles. Il fait un clin d'œil aux Mondes possibles de Lewis (autre œuvre de Jacques Roubaud): "Elle appelle d'un monde possible..."* Il est dialogue muet avec le Journal d'Alix, paroles pour des photos restées sans suite.

L'intertextualité s'étend vers l'extérieur aussi, non seulement par rapport aux modèles classiques: Orphée et Eurydice chez Virgile, puis chez Dante. Conort en voit la référence dans le poème I, 7, "Point vacillant" ("Je ne t'ai pas sauvée de la nuit difficile"/ "Não te salvei da noite difícil"), où apparaissent la nuit, le regard en arrière, la lune. Aussi bien dans IV, 1, le poème dit "Je vais me détourner"/ "Vou me afastar", poème où le critique voit le lieu où la femme apprend au poète qu'il est mortel. Il contient des allusions, des adresses à Georges Perec, à Wittgenstein.

Et Conort conclut: "Les deux touts, arithmétique et référentiel, sont tissus qui, à la manière des appareils que l'on pose sur une blessure, aident au travail cicatriciel". ' Pour le critique, Jacques Roubaud interroge la mort à partir de la représentation de la morte, en ajoutant "l'interrogation qui fut souvent formulée par la poésie moderne et à laquelle chaque poète a apporté sa réponse". Ainsi, le poème de Jacques Roubaud s'il appartient au corpus de la modernité, fonctionne comme un poème à part, qui reflète tel un miroir, l'œuvre passée et l'œuvre à venir du poète.

On ne pourrait pas achever cette présentation sans évoquer le dialogisme de ce recueil, écrit a priori, en réponse à un livre d'Alix Cléo Roubaud, photographe. 
- (PUFF, Jean François. "L'écriture photographique de Quelque chose noir". La Licorne. Revue de langue et littérature française. Presses universitaires de Rennes, 2007:1.)

(Quelque chose noir. op. cit.: 49)

- (Algo: Preto. op. cit.: 53.)

-(VII,6. Quelque chose noir. op. cit.: 114.)

- (Algo: Preto. op. cit.: 113.)

•(III, 9. Quelque chose noir. op. cit.: 57)

-(Algo: Preto. op. cit.: 61.)

Ainsi, de son côté, le critique Jean-François Puff distingue deux phases, deux projets à l'intérieur de l'œuvre roubaldienne. L'une qui "se manifeste comme une mise en mémoire de la tradition poétique... c'est-à-dire d'une poésie conçue comme entrebescar, entrelacement des mots et des sons, réglé par des principes issus de la théorie du rythme qu'il a développée avec Pierre Lusson, ce qui signifie la création de formes nouvelles qui font mémoire de la forme ancienne". Le paradoxe est qu'un nouvel état de poésie à la fois détruit un état de poésie antérieur et se constitue comme sa mémoire.

Le projet de Roubaud commence alors après la rencontre avec Alix Cléo (1979) et se trouve dans le poème "Une logique", III, 5 ("Une sorte de logique pour laquelle tu aurais construit un sens moi une syntaxe, un modèle, des calculs/ Le monde d'un seul, mais qui aurait été deux; pas un solipsisme, un bipsisme..." "* "Uma espécie de lógica para a qual terias construido um sentido eu uma sintaxe, um modelo, cálculos/ O mundo de um só, mas que teria sido dois: não um solipsismo, um biipsismo");" ce projet devait être mené à deux, comme le relate Alix dans son Journal. Dans le Journal, on trouve des séquences comme "la dernière chambre" et "si quelque chose noir". Alix se prend elle-même en photo et par un travail de surimpression afin de "dégager l'âme des choses. Leur double incorporel", l'objet de la photographie se donne comme mémoire, comme "image dans la mémoire". Or, explique le critique, la mort d'Alix abolit le projet d'un travail à deux: ce qui était image la reproduction d'un corps sans vie. ("Il me passait devant les yeux des séquences vitrifiées mais égales, en désaccord violent avec ton immobilité"/ "Passavam diante dos meus olhos sequências vitrificadas mas iguais, em desacordo violento com tua imobilidade". .) Dès lors, de la femme aimée il ne subsiste plus que des photographies où elle s'est photographiée morte, étant vivante. La troisième dimension n'existe plus, seules restent les surfaces. ("Tu n'étais pas blanche et noire plate l'étais-tu?/Tu n'étais pas découpée en rectangle dans le monde" I* "Não eras branca e preta plana. eras?/ Não eras recortada em retângulo no mundo".) $)^{*}$

De ce projet bipsiste, reste une douleur non solipsiste, puisque le poète parvient à s'orienter à l'intérieur de la contradiction entre le projet et l'imprévisible. Selon Conort, "cette image de la photographie, image de l'image, va innerver la totalité du livre, et lui donner son mouvement. Il s'agit de "révéler" progressivement l'étendue du désastre, il s'agit que le négatif, l'inverse de l'image 
quand noir et blanc sont inversés, à la fin, par le bain d'acide, apparaisse, c'est-à-dire en réalité, à la fin “rien”. . L'image nous conduit à Wittgenstein, sur lequel Alix préparait un ouvrage, et, par conséquent, à la logique dont Jacques Roubaud va emprunter la tautologie. "Tu étais morte. et cela ne mentait pas." " Estavas morta. e isso não mentia." Plus loin: "la mort même même. identique à elle-même." I* "a morte mesma mesma. idêntica a ela mesma."” La tautologie énonce que si "la proposition montre ce qu' elle dit", "la tautologie résulte de toutes les propositions: elle ne dit rien". Ce "rien", c'est justement le poème final, l'équivalent de 0 . De plus "dans la tautologie les conditions d'accord avec le monde - les relations de représentation - se suppriment mutuellement de telle sorte qu'elle ne se trouve en aucune relation de représentation à la réalité".* D’où l'impossibilité démontrée de représenter la mort dont on n'aura que l'image de l'image. Pour Conort, la tautologie objectivise la morte, seul moyen de dire la mort.

"Vice logique, selon le Robert, consistant à présenter, comme ayant un sens, une proposition dont le prédicat ne dit rien de plus que le sujet." Vice qui rejoint le vice de Valery, figuré par la méditation.

La mort est identique à elle-même, donc rien n'est dit. En même temps, "la tautologie doit être considérée comme un modèle logique de vérité. La tautologie est inconditionnellement vraie.”* Mais la tautologie, tout modèle de vérité qu'il soit, est aussi le contraire d'un principe de réalité. "La tautologie n'est pas une image de la réalité. Elle ne représente aucun état possible des choses. (...) Dans la tautologie, les conditions d'accord avec le monde - les relations de représentation - se neutralisent les unes les autres, de telle sorte qu' elle ne se trouve en aucune relation de représentation avec la réalité.”*

Or, pour Emmanuel Hocquard, une relation existe entre un nom et la réalité qui se caractérise par le refus de la représentation. L'énoncé tautologique n'explique rien et, en même temps, il dit tout, dit Hocquard. La tautologie en vient à être "une aporie du discours".*

On pourrait continuer indéfiniment à gloser, à expliquer le poème de Roubaud. On peut aussi conclure partiellement que finir sur rien (donc par zéro) n'enlève rien au neuf. La mort "ne dit rien", mais ne peut pas non plus s'abolir ("Je ne peux pas parler de rien, / sans que ce rien,/ Fasse effet d'un retour,/ Et ne cesse qu'avec moi de m'occuper." I* "Não posso falar de nada,/ sem que esse nada,/ Tenha efeito de um retorno,/ E cesse de só comigo me ocupar").*
"(CONORT, Benoït. "Tramer le deuil". op. cit.: 6 .)

•(I, 4. Quelque chose noir. op. cit.: 17)

* (Algo: Preto. op. cit.: 26.)

• (CONORT, Benoït. "Tramer le deuil". op. cit.: 7.)

"(Ibidem: 63.)

(WITTGENSTEIN. Tractatus, 4, 461, cité par Hocquard, Emmanuel, Les Babouches vertes. Marseille: CipM, 2009.)

(WITTGENSTEIN. Tractatus, 4, 462 Hocquard, Emmanuel, Les Babouches vertes. op. cit.)

"(lbidem.)

•(VII, 8. Quelque chose noir. op. cit.: 130).

+(Algo: Preto. op. cit.: 129.) 
On s'en doute, le poème porte l'empreinte de la langue, à travers les formes qui s'y sont inscrites à travers le temps. Le formes de la poésie troubadouresque, d'un côté, mais aussi les formes qui s'y sont inscrites par la traduction, de l'autre.

\section{Conclusion provisoire}

De ce qui vient d'être dit, montré, le traducteur peut en retirer deux conclusions. La première est que le poème est un poème à part dans la production roubaldienne, étant construit de façon assez libre, au gré de l'évocation des images et des paroles.

La deuxième est que le recueil peut être compris et expliqué à l'intérieur de la poétique de Jacques Roubaud, en tant que pierre de touche de son parcours, qui a orienté son travail précédent vers autre chose. Et quand on évoque la poétique de Jacques Roubaud, on veut parler de la poétique française contemporaine qui suit une ligne allant de la tradition vers la rupture. Le poète devient, de ce fait, un ressourceur, ou un pourvoyeur de mots-formes, qu'il convertit dans la langue, qu'il renouvelle du même coup. La langue est toujours le fond, la toile sur laquelle se greffent d'autres formes, d'autres mémoires, d'autres codes. Révéler et innover, voici le programme que Jacques Roubaud, grâce à sa propre mathématique, réussit à accomplir de la meilleure manière tel "il miglior fabbro" de Dante.

Pour en revenir à mon hypothèse de départ, si Jacques Roubaud s'écarte de ses thèses de 1970," (mudei o nous en je) il n'en abandonne pas pour autant les notions de mémoire et de code. Il les complexifie. A partir de et contre Wittgenstein, il construit, selon Stéphane Baquey, un paradoxe: "d'un côté l'expérience de la poésie et avant tout une expérience privée, suscitant des images-mémoire qui sont purement singulières, mais, d'un autre côté, la poésie se

• (BAQUEY, Stéphane. Les possibles de la poésie. op. cit: 638.)

- (Roubaud, Jacques. Poésie, etcetera, ménage. Paris: Stock, 1995: 107.)

(BAQUEY, Stéphane. Les possibles de la poésie. op. cit: 639.) fonde sur des images-langue publiques". * Pour Jacques Roubaud: "la poésie est mémoire externe et mémoire interne".*

Dans les deux cas, toutefois, le poème est nourri de poésie, depuis la poésie japonaise, en passant par la poésie médiévale, la poésie américaine contemporaine, bref la route de la poésie d'aujourd'hui. "La poésie est ainsi une "forme de vie" singulière, survivance d'un art de la mémoire désormais perdu et résistance à cette destruction.”*

Lorsque le poème tend vers la prose, le rythme le récupère. J'ai partout cherché autant que peu se faire à maintenir le rythme, la disposition en vers, les échos, rimes internes et répétitions dans ce texte où la poésie résiste à la poussée de la prose qui, comme disait 
Fernando Pessoa, alias Bernardo Soares, "em prosa é mais difícil de se outrificar"... Le langage apparemment dépouillé du texte original propose des défis au traducteur, des étrangetés, à commencer par les homophonies, des expressions typiquement françaises ("à l'identique", "quelque chose noir"), en plus de l'ice cube émotion.

Au terme de ce parcours, le poète a fini de dire la mort, il l'a apprise, il va mourir, après avoir tenté tous les possibles. Le recueil s'achève sur "Nada", mais c'est un "Rien" désormais posé, écrit.

Ainsi, le poème Nãovida, qui se termine par:

Diz será que eu vou morrer diz

Morrer que eu não saberei mais diz

Ressaca do espaço imperceptível

Vem raspando o instante da sobrevida

Diz da onda de tempo e de quê

De luzes de nuvens de tudo o que faz tudo

Apertando minha mão afastando um pouco a noite

A porta rechaçava luz

Reconheci tua morte e a vi."

"(Algo: Preto. op. cit.: 141.)

Pour revenir à l'intitulé de ma communication, je voudrais ajouter quelques mots en guise de conclusion. Je crois qu'après cette tentative critique sur l'œuvre du poète, à la fois inscrit et novateur dans sa langue, dans sa poétique, on peut comprendre l'amnésie de la traduction brésilienne. Si la mémoire interne (singulière) de quelques poètes brésiliens (Carlos Drummond, Haroldo de Campos, Augusto de Campos, Décio Pignatari, Vinícius de Moraes...) a ouvert des chemins de convergence avec le poète français, à mon avis, tel n'est pas le cas de la mémoire publique, commune. Sauf pour les grandes traductions, à travers celles d'Haroldo de Campos pour la Bible, Homère, la poésie japonaise, celles d'Augusto de Campos pour les troubadours, le sol de la littérature brésilienne, sa mémoire de langue, n'est pas comparable à la mémoire de la littérature française, façonnée depuis longue date. La traduction brésilienne de Quelque chose noir fait surgir donc dans notre langue une poétique inédite, héritière de formes et traditions diverses, absentes dans notre mémoire.

Ce qui fait de Algo: Preto "quelque chose: neuf" dont la traduction - amnésique - s'est efforcée de keep it new. 


\section{Resumo}

Palavras-chave: tradução; poesia francesa; Jacques Roubaud.

A autora apresenta o trabalho do poeta contemporâneo francês Jacques Roubaud e comenta sua tradução brasileira de Quelque chose noir.

Key words: translation; French Poetry; Jacques Roubaud.

Mots-clés: traduction; poésie française; Jacques Roubaud.

\section{Abstract}

The author introduces the work of the contemporary French poet Jacques Roubaud and comments on her Brazilian translation of his Quelque chose noir.

\section{Résumé}

L'auteur présente le travail du poète contemporain français Jacques Roubaud et commente sa traduction du recueil Quelque chose noir. 